\title{
ITERATED FUNCTION SYSTEMS WITH A GIVEN CONTINUOUS STATIONARY DISTRIBUTION
}

\author{
ÖRJAN STENFLO
}

\begin{abstract}
For any continuous probability measure $\mu$ on $\mathbb{R}$ we construct an IFS with probabilities having $\mu$ as its unique measure-attractor.
\end{abstract}

\section{IntRODUCTION}

In 1981 Hutchinson [6] presented a theory of fractals and measures supported on fractals based on iterations of functions.

Let $\left\{\mathbb{R}^{d} ; f_{i}, p_{i}, i=1, \ldots, n\right\}$ be an iterated function system with probabilities (IFSp). That is, $f_{i}: \mathbb{R}^{d} \rightarrow \mathbb{R}^{d}, i=1, \ldots, n$, are functions and $p_{i}$ are associated non-negative numbers with $\sum_{i=1}^{n} p_{i}=1$. If the maps $f_{i}: \mathbb{R}^{d} \rightarrow \mathbb{R}^{d}$ are contractions, i.e. if there exists a constant $c<1$ such that $\left|f_{i}(x)-f_{i}(y)\right| \leq c|x-y|$, for all $x, y \in \mathbb{R}^{d}$, then there exists a unique nonempty compact set $A$ satisfying

$$
A=\cup_{i=1}^{n} f_{i}(A)=\left\{\lim _{k \rightarrow \infty} f_{i_{1}} \circ f_{i_{2}} \ldots \circ f_{i_{k}}(x) ; \quad i_{1} i_{2} i_{3} \ldots \in\{1, \ldots, n\}^{\mathbb{N}}\right\},
$$

for any $x \in \mathbb{R}^{d}$, and a unique probability measure $\mu$, supported on $A$, satisfying the invariance equation

$$
\mu(\cdot)=\sum_{i=1}^{n} p_{i} \mu\left(f_{i}^{-1}(\cdot)\right),
$$

see Hutchinson [6]. The set $A$ is sometimes called the set-attractor, and $\mu$ the measureattractor of the IFSp.

The set-attractor $A$ will have a self-repeating "fractal" appearance if all maps $f_{i}$ are similitudes, and the sets, $f_{i}(A), i=1, \ldots, n$, do not overlap. This leads to the intuition to regard the set-attractor $A$ in (11) as being built up by $n$ (in general overlapping and heavily distorted) "copies" of itself, and the measure-attractor as a "greyscale colouring" of the set-attractor. (Note that the probabilities $p_{i}$ play no role in the definition of $A$.)

In general we can not expect to have a unique set-attractor if the IFS-maps are not assumed to be contractions or more generally if the $\operatorname{limits}_{\lim _{k \rightarrow \infty}} f_{i_{1}} \circ f_{i_{2}} \cdots \circ f_{i_{k}}(x)$ do not exist, with the limit being independent of $x$, for all $i_{1} i_{2} i_{3} \ldots \in\{1, \ldots, n\}^{\mathbb{N}}$, but unique

2000 Mathematics Subject Classification. Primary: 60J05, Secondary: 28A80, 37H99, 60F05, 65C05.

Key words and phrases. Iterated Function Systems, Markov Chain Monte Carlo. 
measure-attractors exist if the limits

$$
\widehat{Z}^{F}\left(i_{1} i_{2} \ldots\right):=\lim _{k \rightarrow \infty} f_{i_{1}} \circ f_{i_{2}} \cdots \circ f_{i_{k}}(x)
$$

exist (with the limit being independent of $x$ ) for almost all $i_{1} i_{2} i_{3} \ldots \in\{1, \ldots, n\}^{\mathbb{N}}$. (Indeed, if the limit in (3) exists a.s. then $\widehat{Z}^{F}$ may be regarded as a random variable, and its distribution $\mu(\cdot):=P\left(\widehat{Z}^{F} \in \cdot\right)$, is then the unique solution to (2) $)$.)

The theory of IFSp has a long pre-history within the theory of Markov chains, starting already with papers in the 30 th by Döblin and others. Let $\left\{X_{k}\right\}_{k=0}^{\infty}$ be the Markov chain obtained by random (independent) iterations with the functions, $f_{i}$, chosen with the corresponding probabilities, $p_{i}$. That is, let $\left\{X_{k}\right\}$ be defined recursively by

$$
X_{k+1}=f_{I_{k+1}}\left(X_{k}\right), k \geq 0,
$$

where $\left\{I_{k}\right\}_{k=1}^{\infty}$ is a sequence of independent random variables with $P\left(I_{k}=i\right)=p_{i}$, independent of $X_{0}$, where $X_{0}$ is some given random variable. (It is well-know that any Markov chain $\left\{X_{k}\right\}$ (with values in $\mathbb{R}^{d}$ ) can be expressed in the form $X_{k+1}=g\left(X_{k}, Y_{k+1}\right)$ where $g: \mathbb{R}^{d} \times[0,1] \rightarrow \mathbb{R}^{d}$ is a measurable function and $\left\{Y_{k}\right\}_{k=1}^{\infty}$ is a sequence of independent random variables uniformly distributed on the unit interval, see e.g. Kifer [7].)

If an IFSp has a unique measure-attractor, $\mu$, then $\mu$ is the unique stationary distribution of $\left\{X_{k}\right\}$, i.e. $\mu$ is the unique probability measure with the property that if $X_{0}$ is $\mu$-distributed, then $\left\{X_{k}\right\}$ will be a (strictly) stationary (and ergodic) stochastic process, see e.g. Elton [5]. Therefore a unique measure-attractor can alternatively also be called a unique stationary distribution.

Under standard average contraction conditions it follows that (3) holds a.s., and the distribution of $X_{k}$ converges weakly to $\mu$ (with exponential rate quantified e.g. by the Prokhorov metric for arbitrary distributions of the initial random variable $X_{0}$ ). Moreover the empirical distribution along trajectories of $\left\{X_{k}\right\}$ converges weakly to $\mu$ a.s., and $\left\{X_{k}\right\}$ obeys a central limit theorem. See e.g. Barnsley et al. [3], Diaconis and Freedman [4], and Stenflo [9] for details and further results. These papers also contains surveys of the literature.

1.1. The inverse problem. The inverse problem is to, given a probability measure $\mu$, find an IFSp having $\mu$ as its unique measure-attractor. This problem is of importance in e.g. image coding where the image, represented by a probability measure, can be encoded by the parameters in a corresponding IFSp in the affirmative cases, see e.g. Barnsley [1]. For an encoding to be practically useful it needs to involve few parameters and the distribution of $X_{k}$ needs to converge quickly to equilibrium (a property ensured by average contractivity properties of the functions in the IFSp) for arbitrary initial distributions of $X_{0}$.

It is possible to construct solutions to the inverse problem in some very particular cases using Barnsley's "collage theorem", see [1] containing exciting examples of e.g. ferns and clouds (interpreted as probability measures on $\mathbb{R}^{2}$ ) and their IFSp encodings, 
but typically it is very hard to even find approximate solutions to the inverse problem for general probability measures on $\mathbb{R}^{d}$.

In this paper we present a (strikingly simple) solution to the inverse problem for continuous probability measures on $\mathbb{R}$.

\section{MAin RESUlT}

In order to present our solution to the inverse problem for continuous probability measures on $\mathbb{R}$, recall the following basic facts used in the theory of random number generation;

Let $\mu$ be a probability measure on $\mathbb{R}$, and let $F(x)=\mu((-\infty, x])$ denote its distribution function. The generalised inverse distribution function is defined by

$$
F^{-1}(u)=\inf _{x \in \mathbb{R}}\{F(x) \geq u\}, \quad 0 \leq u \leq 1
$$

and satisfies $F^{-1}(F(x)) \leq x$ and $F\left(F^{-1}(u)\right) \geq u$ and therefore

$$
F^{-1}(u) \leq x \quad \text { if and only if } \quad u \leq F(x)
$$

From this it follows that if $U \in U(0,1)$, i.e. if $U$ is a random variable uniformly distributed on the unit interval, then $F^{-1}(U)$ is a $\mu$-distributed random variable. This basic property reduces the problem of simulating from an arbitrary distribution on $\mathbb{R}$, to the problem of simulating uniform random numbers on the unit interval.

We say that $\mu$ is continuous if $F$ is continuous. Note that $\mu(\{x\})=0$ for any $x \in \mathbb{R}$ for continuous probability measures in contrast with discrete probability measures where $\sum_{x \in S} \mu(\{x\})=1$ for some countable set $S$.

If $\mu$ is continuous then $F\left(F^{-1}(u)\right)=u$, for $0<u<1$. This property is crucial for the following theorem;

Theorem 1. A continuous distribution, $\mu$, on $\mathbb{R}$ with distribution function, $F$, is the measure-attractor of the IFS with monotone maps $f_{i}(x):=F^{-1} \circ u_{i} \circ F(x)$, for any $x$ with $F(x)>0$, and probabilities $p_{i}=1 / n$, where $u_{i}(u)=u / n+(i-1) / n, 0 \leq u \leq 1$, $i=1,2, \ldots, n$, for any $n \geq 2$.

Proof. The Markov chain generated by $u_{i}(x)=x / n+(i-1) / n, i=1,2, \ldots, n$, chosen with equal probabilities has the uniform distribution on the unit interval as its unique stationary distribution. That is, if $\left\{I_{k}\right\}_{k \geq 1}$ is a sequence of independent random variables, uniformly distributed on $\{1,2, \ldots, n\}$, then

$$
Z_{k}^{U}(x)=u_{I_{k}} \circ \cdots \circ u_{I_{1}}(x), \quad Z_{0}^{U}(x)=x
$$

is a Markov chain starting at $x \in[0,1]$ having the uniform distribution as its unique stationary distribution. This can be seen by observing that $Z_{k}^{U}(x)$ has the same distribution as the reversed iterates

$$
\widehat{Z}_{k}^{U}(x)=u_{I_{1}} \circ \cdots \circ u_{I_{k}}(x), \quad \widehat{Z}_{0}^{U}(x)=x,
$$


for any fixed $k$, and the reversed iterates $\widehat{Z}_{k}^{U}(x)$ converges almost surely to the $U(0,1)$ distributed random variable, $\widehat{Z}^{U}$, where the $k: t h$ digit in the base $n$ expansion of $\widehat{Z}^{U}$ is given by $I_{k}-1$.

If $\widehat{Z}^{F}$ denotes the limit of the reversed iterates of the system with $f_{i}$ chosen with probability $1 / n$, then

$$
\begin{aligned}
\widehat{Z}^{F} & :=\lim _{k \rightarrow \infty} \widehat{Z}_{k}^{F}(x):=\lim _{k \rightarrow \infty} f_{I_{1}} \circ \cdots \circ f_{I_{k}}(x) \\
& =\lim _{k \rightarrow \infty} F^{-1} \circ u_{I_{1}} \circ F \circ F^{-1} \circ u_{I_{2}} \circ F \circ F^{-1} \circ u_{I_{k}} \circ F(x) \\
& =\lim _{k \rightarrow \infty} F^{-1} \widehat{Z}_{k}^{U}(F(x))=F^{-1}\left(\widehat{Z}^{U}\right) \quad \text { a.s. },
\end{aligned}
$$

where the last equality holds since $F^{-1}(x)$ is non-decreasing, and since a monotone function can have at most a countable set of discontinuity points in its domain, it follows that $F^{-1}(x)$ is continuous for a.a. $x \in[0,1]$ w.r.t. to the Lebesgue measure.

From the above it follows that

$$
P\left(\widehat{Z}^{F} \leq y\right)=P\left(F^{-1}\left(\widehat{Z}^{U}\right) \leq y\right)=P\left(\widehat{Z}^{U} \leq F(y)\right)=F(y) .
$$

Remark 1. If $X$ is a continuous $\mu$-distributed random variable, then $F\left(F^{-1}(u)\right)=u$, so $F(X) \in U(0,1)$. This contrasts the case when $X$ is discrete where $F(X)$ will also be discrete, so we cannot expect Theorem 1$]$ to generalise to discrete distributions.

If an IFS $\left\{\mathbb{R}, f_{i}, p_{i}, i=1, \ldots, n\right\}$, has a continuous measure-attractor $\mu$ being the distribution of the a.s. limit of the reversed iterates, and the distribution function $F$ of $\mu$ satisfies $F^{-1}(F(x))=x$, for any $x \in \mathbb{R}$, with $0<F(x)<1$, then, similarly, the IFS $\left\{[0,1], u_{i}, p_{i}, i=1, \ldots, n\right\}$, with $u_{i}(u):=F \circ f_{i} \circ F^{-1}(u), 0<u<1$, has the $U(0,1)$ distribution as its unique stationary distribution. This is the case for absolutely continuous probability distributions $\mu$ if $F$ is strictly increasing.

Remark 2. From Theorem 1 it follows that any continuous probability distribution on $\mathbb{R}$ can be approximated by the empirical distribution of a Markov chain $\left\{X_{k}\right\}$ on $\mathbb{R}$ generated by an IFSp with trivial "randomness" generated by e.g. by a coin or a dice.

Remark 3. Theorem 1 may be used to represent a continuous probability measure $\mu$ on $\mathbb{R}$ by the functions suggested in the theorem. Note that there exist many iterated function systems with probabilities generating the same Markov chain, see e.g. Stenflo [8], so in particular it follows than an IFSp representation of a continuous probability measure on $\mathbb{R}$ is not unique. The given IFSp representation suggested by Theorem 1 (for a given $n \geq 2$ ) is good in the sense that the generated Markov chain converges quickly to the given equilibrium making it possible to quickly simulate it. If the suggested IFSp representation cannot be described in terms of few parameters then it might make sense to consider an approximate representation by approximating the IFS functions with functions described by few parameters e.g. by using Taylor expansions. 
Remark 4. From Theorem 1 it follows that if $\mu$ is a continuous probability measure on $\mathbb{R}$ being the measure-attractor of $\left\{\mathbb{R} ; f_{i}, p_{i}, i=1, \ldots, n\right\}$, with $p_{i} \neq 1 / n$ for some $n$, then there exists another IFSp with uniform probabilities having $\mu$ as its measure-attractor.

Example 1. Suppose $F$ is a distribution function satisfying

$$
F(1-x)=1-F(x),
$$

and

$$
F(x) / 2=F(a x+b), \text { for all } 0 \leq x \leq 1,
$$

where $0 \leq b \leq 1 / 2,0 \leq a+b \leq 1 / 2$, and $a \neq 0$.

Then

$$
F(x) / 2+1 / 2=1-F(1-x) / 2=1-F(a(1-x)+b)=F(a x+1-a-b) .
$$

Thus random iterations with the maps $f_{1}(x)=a x+b$, and $f_{2}(x)=a x+1-a-b$ chosen with equal probabilities generates a Markov chain with stationary distribution $\mu$ having distribution function $F$.

The case $a=1 / 3$ and $b=0$ corresponds to $F$ being the distribution function of the uniform probability measure on the middle-third Cantor set (the Devil's staircase).

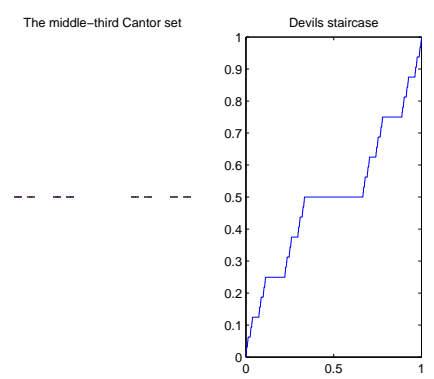

The Cantor set is the set-attractor of the IFSp $\left\{\mathbb{R} ; f_{1}(x)=x / 3, f_{2}(x)=x / 3+2 / 3, p_{1}=1 / 2, p_{2}=1 / 2\right\}$ and the distribution function of its measure-attractor (the uniform distribution on the Cantor set) is an increasing continuous function with zero derivative almost everywhere, with $F(0)=0$ and $F(1)=1$ popularly known as the "Devil's staircase".

Example 2. Let $\mu$ be the probability measure with triangular density function

$$
d(x)=\left\{\begin{array}{ll}
x & 0 \leq x \leq 1 \\
2-x & 1 \leq x \leq 2
\end{array} .\right.
$$

Then $\mu$ is the unique stationary distribution of the Markov chain generated by random iteration with the functions

$$
f_{1}(x)= \begin{cases}\frac{x}{\sqrt{2}} & 0 \leq x \leq 1 \\ \sqrt{2 x-\frac{x^{2}}{2}-1} & 1 \leq x \leq 2\end{cases}
$$


and

$$
f_{2}(x)=\left\{\begin{array}{ll}
2-\sqrt{1-\frac{x^{2}}{2}} & 0 \leq x \leq 1 \\
2-\sqrt{2-2 x+\frac{x^{2}}{2}} & 1 \leq x \leq 2
\end{array},\right.
$$

chosen uniformly at random.
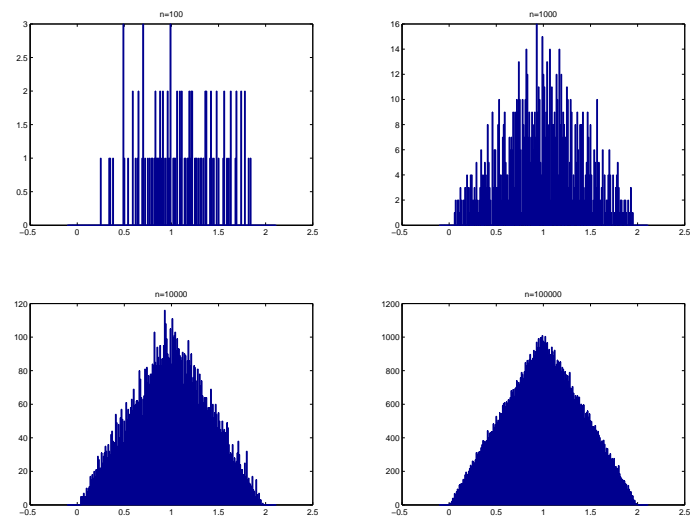

Histograms of the first $n$ points in a simulated random trajectory of the Markov chain. The empirical distribution along a trajectory converges weakly to the stationary triangular-distribution with probability one.

Example 3. The distribution function for the exponential distribution with expected value $\mu=\lambda^{-1}, \lambda>0$, satisfies $F(x)=1-e^{-\lambda x}, x \geq 0$. A Markov chain generated by random iterations with the two maps $f_{1}=f_{1}^{\mu}$ and $f_{2}=f_{2}^{\mu}$ defined as in Theorem 1 ] has the exponential distribution with expected value $\mu$ as its stationary distribution. We can construct interesting "new" distributions by altering such Markov chains in various ways, e.g. by altering the application of two IFSs corresponding to different parameter values. A result of such a construction is shown in the figure.
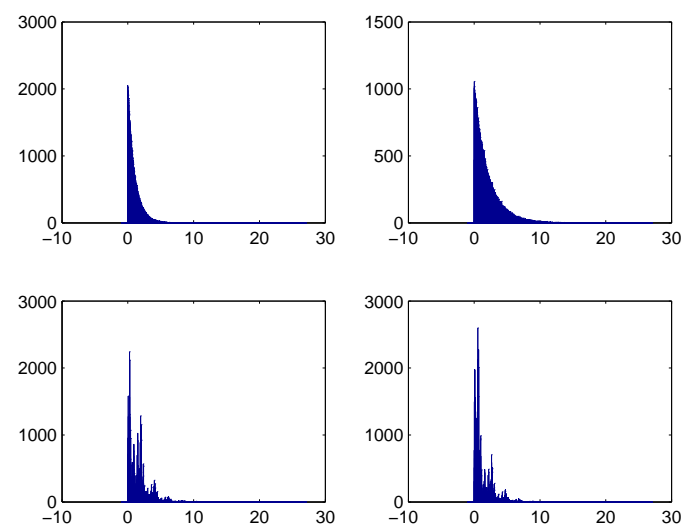
The upper figures are histograms of the first 200000 points in simulations of a trajectory of a Markov chain generated by random iterations with the two maps $f_{1}=f_{1}^{\mu}$ and $f_{2}=f_{2}^{\mu}$ defined as in Theorem 1 corresponding to the choices $\mu=1$ in the left hand figure and $\mu=2$ in the righthand figure respectively.

The lower figures are histograms corresponding to trajectories of Markov chains formed by random iterations with the maps $g_{1}(x)=f_{1}^{2}\left(f_{1}^{1}(x)\right), g_{2}(x)=f_{1}^{2}\left(f_{2}^{1}(x)\right), g_{3}(x)=$ $f_{2}^{2}\left(f_{1}^{1}(x)\right), g_{4}(x)=f_{2}^{2}\left(f_{2}^{1}(x)\right)$ and $h_{1}(x)=f_{1}^{1}\left(f_{1}^{2}(x)\right), h_{2}(x)=f_{1}^{1}\left(f_{2}^{2}(x)\right), h_{3}(x)=$ $f_{2}^{1}\left(f_{1}^{2}(x)\right), h_{4}(x)=f_{2}^{1}\left(f_{2}^{2}(x)\right)$ respectively, where in both cases the functions are chosen uniformly at random.

Remark 5. The distributions constructed in the lower figures in the example above are 1 -variable mixtures of the exponential distributions with expected values $\mu=1$, and $\mu=2$ respectively. We can, more generally, for any integer $V \geq 1$, generate $V$-variable mixtures between continuous distributions. See Barnsley et al. [2] and [3] for more on the theory of $V$-variable sets and measures.

\section{REFERENCES}

[1] M. F. Barnsley. Fractals everywhere. Second edition, Academic Press, Boston, MA, 1993.

[2] M. F. Barnsley, J. E. Hutchinson and Ö. Stenflo. A fractal valued random iteration algorithm and fractal hierarchy Fractals, 13(2):111-146, 2005.

[3] M. F. Barnsley, J. E. Hutchinson and Ö. Stenflo. $V$-Variable fractals: Fractals with partial self similarity. Adv. Math., 218(6):2051-2088, 2008.

[4] P. Diaconis and D. Freedman. Iterated random functions. SIAM Rev., 41(1):45-76 (electronic), 1999.

[5] J. H. Elton. An ergodic theorem for iterated maps. Ergodic Theory Dynam. Systems, 7(4):481-488, 1987.

[6] J. E. Hutchinson. Fractals and self-similarity. Indiana Univ. Math. J., 30(5):713-747, 1981.

[7] Y. Kifer. Ergodic theory of random transformations., Birkhäuser, Boston, MA, 1986.

[8] Ö. Stenflo. Ergodic theorems for Markov chains represented by iterated function systems. Bull. Polish Acad. Sci. Math. 49(1):27-43, 2001.

[9] Ö. Stenflo. A survey of average contractive iterated function systems. To appear in J. Difference Equ. Appl.

E-mail address: stenflo@math.uu.se

Department of Mathematics, Uppsala University, 75106 Uppsala, Sweden 